\title{
Mass spectrometry reveals protein kinase CK2 high-order oligomerization via the circular and linear assembly
}

\author{
Wei-Guang Seetoh ${ }^{\star}$, Daniel Shiu-Hin Chan, Dijana Matak-Vinković, and Chris Abell ${ }^{\star}$ \\ Department of Chemistry, University of Cambridge, Lensfield Road, Cambridge, CB2 1EW, \\ United Kingdom
}

\section{Abstract}

CK2 is an intrinsically active protein kinase that is crucial for cellular viability. However, conventional kinase regulatory mechanisms do not apply to CK2 and its mode of regulation remains elusive. Interestingly, CK2 is known to undergo reversible ionic-strength dependent oligomerization. Furthermore, a regulatory mechanism based on autoinhibitory oligomerization has been postulated based on the observation of circular trimeric oligomers and linear CK2 assemblies in various crystal structures. Here, we employ native mass spectrometry to monitor the assembly of oligomeric CK2 species in an ionic strength-dependent manner. A subsequent combination of ion mobility spectrometry-mass spectrometry and hydrogen-deuterium exchange mass spectrometry techniques was used to analyze the conformation of CK2 oligomers. Our findings support ionic strength-dependent CK2 oligomerization, demonstrate the transient nature of the $a / \beta$ interaction, and show that CK2 oligomerization proceeds via both the circular and linear assembly.

Protein kinase CK2 is a pleiotropic, ubiquitous, acidophilic and highly conserved serine/ threonine kinase that is essential for cellular viability.1,2 CK2 is involved in various cellular processes such as cell cycle control, cellular differentiation and proliferation, the circadian rhythm, apoptosis and gene expression.1,3 In humans, CK2 exists as a heterotetrameric holoenzyme $\left(a_{2} / \beta_{2}\right)$ composed of two catalytic $a$-subunits (denoted as CK2 $a$ or $a$ ) attached to a central, regulatory dimer of $\beta$-subunits (denoted as $\mathrm{CK} 2 \beta$ or $\beta_{2}$ ). 4

A unique feature of CK2 $a$ is its constitutively active nature, both in its apo and holoenzyme form, due to its activation segment being maintained in an active conformation through interaction with its $\mathrm{N}$-terminal region.4-6 Upon interaction with CK2 $\beta$, CK2 $a$ does not undergo any significant structural changes except at the $a / \beta$ interfacial region, and therefore retains its intrinsic catalytic capacity.7 CK2 $\beta$ is not an on-off regulator of the catalytic activity of CK $2 a$. Instead, CK2 $\beta$ alters other properties of CK $2 a$, such as its thermostability, substrate specificity and ability to attach and penetrate cell membranes.7-9 Indeed, conventional kinase regulatory mechanisms such as phosphorylation, dephosphorylation, or second messenger binding are not observed for CK2.10 The regulation of CK2 activity therefore remains poorly defined.

*Corresponding authors: wgs25@cam.ac.uk; ca26@cam.ac.uk. 
Interestingly, CK2 has been shown to form high-order oligomers or aggregates in solutions with low ionic strength.11-13 Crystal structures of CK2 suggest that oligomerization is driven by electrostatic interactions between the acidic loop of CK2 $\beta$ with the positivelycharged substrate-binding region of CK2 $a$ from an adjacent CK2 heterotetramer, representing a structural determinant for an autoinhibitory mechanism of CK2 regulation. 14-16 Additionally, crystal structures have revealed two modalities by which CK2 oligomerization could proceed. Circular trimeric oligomers 14 were observed in crystalline assemblies of CK2 (1JWH4 and 4DGL16), whereas relatively linear polymers were observed in monoclinic structures (4MD7-917 and 4NH115). Here, we present mass spectrometry (MS) evidence to support ionic strength-dependent CK2 oligomerization, demonstrate the transient nature of the $a / \beta$ interaction, and show that CK2 oligomerization occurs via both the circular and linear assemblies.

Native mass spectra of CK2 $a$ and CK2 $\beta$ were acquired individually at $5 \mu \mathrm{M}$ in $0.50 \mathrm{M}$ ammonium acetate under non-denaturing conditions by nano-electrospray ionization-mass spectrometry (nESI-MS) on a hybrid ion mobility-time-of-flight Synapt HD mass spectrometer. Instrument conditions were carefully optimized to maximize ion desolvation while preserving the structural integrity of non-covalent protein complexes (Supplementary Methods). Both CK2 $a$ (Figure 1a) and CK2 $\beta$ (Figure 1b) produced well-resolved charge state series corresponding to a predominantly monomeric CK2 $\alpha$ and dimeric CK2 $\beta$ state, consistent with published structural data.4,18 There was good agreement between experimental masses and masses calculated from the protein sequences (Supplementary Table 1).

$\mathrm{CK} 2 \beta$ was incubated with CK2 $a$ at 2-fold molar ratio in ammonium acetate solutions with varying ionic strengths $(0.40-0.75 \mathrm{M})$ (Figure $1 \mathrm{c})$. At $0.75 \mathrm{M}$ ammonium acetate, only the monomeric CK2 heterotetramer, $\left(a_{2} / \beta_{2}\right)_{1}$, was observed. As the ionic strength of the solution decreased from $0.75 \mathrm{M}$ to $0.40 \mathrm{M}$ ammonium acetate, two charge state series with higher $\mathrm{m} / \mathrm{z}$ values compared to the monomeric CK2 heterotetramer, $\left(a_{2} / \beta_{2}\right)_{1}$, were observed. These series were assigned as a dimer $\left[\left(a_{2} / \beta_{2}\right)_{2}, 251 \mathrm{kDa}\right]$ and trimer $\left[\left(a_{2} / \beta_{2}\right)_{3}, 379 \mathrm{kDa}\right]$ of the CK2 heterotetramer. This indicates that the formation of higher-order oligomers is favored in solutions of low ionic strength. Importantly, the observation of the CK2 dimer provides the first evidence of its biochemical existence and supports postulated models of trans-phosphorylation of the CK2 $\beta \mathrm{N}$-termini, in which two CK2 monomers interact to mutually phosphorylate Ser 2 of CK2 $\beta .19,20$ Unfortunately, CK2 oligomerization at lower ammonium acetate concentrations could not be investigated due to CK2 $a$ precipitation.

As CK2 $\beta$ was known to be synthesized in excess of CK2 $\alpha$ in cells and confer thermostability to CK2 $a, 7,21$ CK2 $a$ was incubated with CK2 $\beta$ at 1.2 -fold molar ratio. This enabled the investigation of CK2 oligomerization to as low as $0.20 \mathrm{M}$ ammonium acetate without causing precipitation. At higher concentrations of ammonium acetate $(0.60-0.75$ $\mathrm{M})$, no oligomers were detected, with the monomeric CK2 heterotrimer $\left(a_{1} / \beta_{2}\right)$ and heterotetramer $\left(a_{2} / \beta_{2}\right)$ being the only species observed (Figure 1d). Detectable levels of CK2 oligomerization are observed at $0.50 \mathrm{M}$ ammonium acetate, and at $0.20 \mathrm{M}$ ammonium acetate, almost all monomeric CK2 holoenzymes are assembled into various higher-order species. As the ammonium acetate concentration of the solution was decreased from 0.60 to 
$0.20 \mathrm{M}$, the population of monomeric CK2 diminished, while multiple high-order oligomeric species appeared. These included the dimeric $\left[\left(a_{2} / \beta_{2}\right)_{2}, 251 \mathrm{kDa}\right]$ and trimeric $\left[\left(a_{2} / \beta_{2}\right)_{3}, 379 \mathrm{kDa}\right]$ complexes of the CK2 heterotetramer that were observed in the experiments with excess CK2 $a$ (Figure 1c), as well as species observed in different possible states of oligomerization that were putatively assigned as [ $\left.\left(a_{1} / \beta_{2}\right)_{2}, 172 \mathrm{kDa}\right],\left[\left(a_{1} / \beta_{2}\right)_{2}+\right.$ $\left.\beta_{2}, 217 \mathrm{kDa}\right],\left[\left(a_{1} / \beta_{2}\right)_{3}, 258 \mathrm{kDa}\right],\left[\left(a_{2} / \beta_{2}\right)_{2}+\beta_{2}, 298 \mathrm{kDa}\right],\left[\left(a_{1} / \beta_{2}\right)_{3}+\beta_{2}, 305 \mathrm{kDa}\right]$, $\left[\left(a_{2} / \beta_{2}\right)_{2}+a_{1} / \beta_{2}, 338 \mathrm{kDa}\right]$ and $\left[\left(a_{1} / \beta_{2}\right)_{4}, 343 \mathrm{kDa}\right]$. The diversity of oligomeric species with different compositions highlights the transient nature of the $a / \beta$ interaction postulated from structural analysis of the first CK2 holoenzyme crystal structure (PDB: 1JWH4). More importantly, the multiplicity of oligomeric species supports "jumping-out-of-the-catalyticbox" strategies of inhibiting CK2 activity through targeting the unique dynamic assembly of CK2, which may generate antagonists with greater specificity than ATP-competitive inhibitors. 22

In $0.20 \mathrm{M}$ ammonium acetate (Figure 1d), we observe a tetrameric CK2 species, $\left(a_{1} / \beta_{2}\right)_{4}$, potentially resembling the maximally active ring-like structures of Drosophila CK2 with the $\left(a_{2} / \beta_{2}\right)_{4}$ composition.13 Ring-like structures and monomeric CK2 were the dominant species in $0.2 \mathrm{M}$ and $0.4 \mathrm{M} \mathrm{NaCl}$, respectively, in the study of Drosophila CK2 oligomerization.13 However, our native MS results showed that CK2 exists as a mixture of oligomeric species in both $0.2 \mathrm{M}$ (Figure 1d) and $0.4 \mathrm{M}$ (Figure 1c and 1d) ammonium acetate conditions. These discrepancies could be attributed to the use of different experimental conditions. The observation that the ratio of the monomeric CK2 heterotrimer, $\left(a_{1} / \beta_{2}\right)_{1}$, to heterotetramer, $\left(a_{2} / \beta_{2}\right)_{1}$, complexes increases with decreasing ammonium acetate concentration indicates that the binding stoichiometry between CK2 $\alpha$ and CK2 $\beta$ is influenced by ionic strength. Interestingly, the observation of the monomeric CK2 heterotrimer, $\left(a_{1} / \beta_{2}\right)_{1}$, at intermediate ionic strength (0.30-0.60 M ammonium acetate) corresponds to PISA's ('Proteins, Interfaces, Structures and Assemblies' service, European Bioinformatics Institute) 23 predicted dissociation modality of $a_{2} / \beta_{2}$ to $a_{1} / \beta_{2}$ and $a$ for 1JWH4 and 4DGL, 16 in which trimeric rings are observed,14 suggesting that CK2 oligomerization occurs via the circular trimeric assembly. It is questionable whether monomeric CK2 dissociates from the heterotetrameric to heterotrimeric form (i.e. $a_{2} / \beta_{2}$ to $\left.a_{1} / \beta_{2}\right)$ as the ionic strength decreases, given the strong affinity of interaction $\left(\mathrm{K}_{\mathrm{D}}=5-12\right.$ $\mathrm{nM}$ ) between CK2 $a$ and CK2 $\beta .7,24$ However, our native MS data reveals the transient character of the $a / \beta$ interaction. The co-existence of $\left(a_{2} / \beta_{2}\right)_{\mathrm{n}}$ with $\left(a_{1} / \beta_{2}\right)_{\mathrm{n}}$ (Figure 1d) indicates that the two CK2 $a$-binding sites on CK2 $\beta$ does not necessarily reach full occupancy despite the strong affinity of interaction between CK $2 a$ and CK2 $\beta$. When the ionic strength decreases, one of the $a$-subunits could reversibly dissociate from the CK2 heterotetramer $\left(a_{2} / \beta_{2}\right)$ in order to form even more stable oligomeric species, confirming the transient nature of the CK2 holoenzyme as previously postulated.4

The proximity between the acidic loop of CK2 $\beta$ and the basic regions of CK2 $a$ (basic cluster at $a \mathrm{C}$ helix and $\mathrm{P}+1$ loop) responsible for downregulating kinase activity has been suggested by published mutational and enzymological studies.25 Furthermore, the crystal structures of CK2 in the hexagonal (PDB: 1JWH4, 4DGL16) and monoclinic crystal packing (PDB: 4MD7-9,17 4NH115) have demonstrated this proximity. To investigate whether CK2 oligomerization is driven by the electrostatic interaction between the 
negatively-charged acidic loop of CK2 $\beta$ with the positively charged regions of CK2 $a$ from a neighboring CK2 heterotetramer, as inferred from $X$-ray crystal structures, 4,16 mutagenesis experiments were performed. A CK2 $\beta$ mutant with three glutamate residues of the acidic loop mutated to alanine (CK2 $\beta^{\mathrm{E} 60 \mathrm{~A} / \mathrm{E} 61 \mathrm{~A} / \mathrm{E} 63 \mathrm{~A}}$ ) formed mainly monomeric CK2 heterotetramer, $\left(a_{2} / \beta^{\mathrm{E} 60 \mathrm{~A} / \mathrm{E} 61 \mathrm{~A} / \mathrm{E} 63 \mathrm{~A}}{ }_{2}\right)_{1}$, and only a minor population of dimeric CK2 heterotetramer, $\left(a_{2} / \beta^{\mathrm{E} 60 \mathrm{~A} / \mathrm{E} 61 \mathrm{~A} / \mathrm{E} 63 \mathrm{~A}}\right)_{2}$, was detected (Supplementary Figure 1). The lack of $\mathrm{CK} 2$ oligomer formation for $\mathrm{CK} 2 \beta^{\mathrm{E} 60 \mathrm{~A} / \mathrm{E} 61 \mathrm{~A} / \mathrm{E} 63 \mathrm{~A}}$ compared to when wild-type CK2 $\beta$ is used can be explained by the elimination of charges on its acidic loop, which compromises electrostatic interactions with the basic regions of CK2 $a$. These results validate structural observations that electrostatic interactions between the acidic loop and the basic regions of CK2 $a$ drive CK2 oligomerization.14,15

While native MS enabled the characterization of the stoichiometry of oligomeric CK2 species, it does not provide information about the conformational state of the complexes. For instance, $\left(a_{2} / \beta_{2}\right)_{3}$ could exist either in a linear or circular trimeric conformation, or as a mixed population of both. Hence, ion mobility-spectrometry coupled to nESI-MS (IMSMS) was used to examine the conformational state of the oligomers. Under non-denaturing conditions, drift times were recorded for four charge states of the monomeric, dimeric, and trimeric CK2 heterotetramer (Figure 2a) on the Synapt HD mass spectrometer interfaced with a traveling-wave (TW) IMS device, and then converted into collision cross sections (CCS) (Figure 2b) by calibration with protein standards. Table 1 shows a comparison between the experimental and theoretical CCS of various CK2 oligomers. Theoretical CCS were calculated from the corresponding X-ray crystal structures using the projection approximation (PA) method implemented in DriftScope 2.5.

The experimentally determined CCS for monomeric CK2 averaged across four charge states $\left(7,420 \pm 26 \AA^{2}\right)$ is similar to the theoretical CCS calculated from four different monomeric CK2 crystal structures $\left(6,747-6,906 \AA^{2}\right)$. Meanwhile, the average experimentally determined CCS for dimeric CK2 is $11,820 \pm 90 \AA^{2}$. This is closer to the theoretical CCS values of a linear CK2 dimer (12,010 $\AA^{2}$ for 4 MD917 and 12,180 $\AA^{2}$ for 4NH115) compared to a crescent-shaped CK2 dimer (12,420 $\AA^{2}$ for 1 JWH4 and $12,380 \AA^{2}$ for 4DGL16), which represents an intermediate form of a fully formed circular trimeric ring. The CK2 dimer adopts the linear conformation, resembling models for transphosphorylation of the CK2 $\beta \mathrm{N}$-termini derived from the symmetrical docking of two monomeric CK2.19,20 In addition, the detection of the CK2 dimer in the linear conformation fits to the linear dimer observed in the monoclinic crystal packing of 4NH1.15 On the other hand, the average experimental CCS for trimeric CK2 of $15,470 \pm 245 \AA^{2}$ is closer to the theoretical CCS of a trimeric CK2 ring $\left(16,190 \AA^{2}\right.$ for $1 \mathrm{JWH} 4$ and $16,590 \AA^{2}$ for 4DGL16) than a linear CK2 trimer (17,180 $\AA^{2}$ for 4MD917 and 17,670 $\AA^{2}$ for 4NH115). Taken together, the IMS-MS data supports the formation of both linear and circular CK2 oligomers, with the dimer preferring to exist in a linear conformation, and the trimer favoring a ring conformation.

In order to further examine the conformational state of CK2 oligomers, hydrogen-deuterium exchange mass spectrometry (HDX-MS) experiments were performed. CK2 complexes were incubated in either low salt conditions $(0.20 \mathrm{M} \mathrm{NaCl})$, in which $\mathrm{CK} 2$ would be 
expected to exist mostly in oligomeric form, or high salt conditions $(0.75 \mathrm{M} \mathrm{NaCl})$, in which monomeric CK2 would be expected to be the major species. By studying the relative differences in deuterium uptake at different ionic strengths, HDX-MS could inform on protein structure changes during oligomerization, and enable characterization of the oligomeric conformation. The difference in deuterium exchange was analyzed with respect to both CK2 $a$ and CK2 $\beta$, with protein sequence coverage of $94.6 \%$ and $96.5 \%$, respectively (Supplementary Figure 2).

Two regions in CK2 $a$, spanning residues 110-130 and 181-201, respectively, experienced small but significant decreases in deuterium uptake upon transition from high salt (monomeric CK2) to low salt (oligomeric CK2) conditions (Figure 3a). This indicates that those regions of CK2 $a$ become more occluded during oligomerization. As residues 181-201 correspond to the substrate binding region $(\mathrm{P}+1$ loop) on $\mathrm{CK} 2 a$, the greater protection of this region under low salt conditions can be attributed to the interaction of those residues with the acidic loop of a CK2 $\beta$ subunit from a neighboring CK2 holoenzyme during oligomerization. An analysis of CK2 crystal structures showed that this pattern of deuterium exchange supports the formation in solution of circular trimeric CK2 (Figure 3b), in which the acidic loop of $\mathrm{CK} 2 \beta$ is directed to the basic $\mathrm{P}+1$ loop of $\mathrm{CK} 2 a$.14 Furthermore, the increased protection of the peptide region spanning residues 110-130 of CK2 $a$ is consistent with the circular trimeric mode of oligomerization. An X-ray crystal structure composed of circular, trimeric CK2 assemblies (PDB: 4DGL16) showed that those trimers were stacked upon one another with an offset of $60^{\circ}$ between adjacent trimers (Figure 3c), forming a filamentous assembly.16 A close examination of a trimer-trimer interface reveals a small, symmetric intermolecular contact between regions 110-130 of CK2 $a$ subunits belonging to adjacent trimeric rings (Figure 3d). In 4DGL, an inter-trimeric ring interaction was observed, in which the $\mathrm{C}$-terminus of $\mathrm{CK} 2 \beta$ arising from one trimeric ring makes contact with the $\mathrm{C}$-terminal domain of a CK2 $a$ subunit originating from an adjacent layer of trimeric ring, and eventually terminates in the CK2 a ATP-binding site. 16 This interaction was suggested to be crucial for piling organization, but not observed in $1 \mathrm{JWH}$ due to poorly-defined electron density of the last ten residues in the CK2 $\beta$ C-terminal tail.4,16 However, despite using C-terminally truncated constructs of CK2 $\alpha$ and CK2 $\beta$ in our study, the stacking interaction could still be observed in solution, suggesting that the $\mathrm{C}$-terminal tail of CK2 $\beta$ may not be essential for stacking. Overall, the decreased deuterium uptake of peptide region 110-130 of CK2 $a$ subunits upon transition to a low salt buffer provides evidence of a stacked trimeric ring mode of CK2 oligomerization.

Two peptide regions in CK2 $\beta$ experienced significant decreases in deuterium uptake upon transition from high salt to low salt conditions (Figure 4a). The greater protection of this region 40-65, which includes the CK $2 \beta$ acidic loop, under low salt conditions can be attributed to the interaction of the acidic residues with the basic region of CK2 $a$. The region spanning residues $148-164$ of CK2 $\beta$ supports the formation of linear oligomers in solution. Examination of the crystalline assembly in 4NH115 reveals that the residues 148-164 of CK2 $\beta$ from one holoenzyme makes an intermolecular contact with a CK2 $a$ subunit originating from a neighboring holoenzyme (Figure 4b). In all, our HDX-MS and IMS-MS data support the existence of circular and linear assemblies of CK2 oligomers. 
Significant in vitro biochemical data have demonstrated the ability of the CK2 holoenzyme to undergo reversible, ionic strength-dependent oligomerization, which is further modulated by other factors such as the presence of polycations, $\mathrm{Mg}^{2+}$ concentration, $\mathrm{pH}$ and temperature.11-13 It is widely recognized that CK2 can be maintained in a relatively monodisperse and soluble form at $0.5 \mathrm{M}$ or higher concentrations of salt, below which CK2 begins to aggregate.15,16 Hence, a certain proportion of CK2 would be expected to be present as oligomers in a physiological environment. These oligomers have been characterized in vitro and can be broadly divided into inactive filamentous and maximally active ring-like aggregates.13 In fact, there now exists evidence for CK2 holoenzyme aggregation in cells, highlighting a unique regulatory mechanism for eukaryotic protein kinases. 26

Our mass spectrometric results confirm the ionic strength-dependent nature of CK2 oligomerization. Crucially, our native MS experiments reveal the transient nature of the $a / \beta$ interaction and the dynamic assembly of CK2, as shown by the observation of diverse oligomeric species with different compositions. The observation that the CK2 $a / \mathrm{CK} 2 \beta$ stoichiometry varies with changing environmental conditions supports the development of inhibitors of CK2 oligomerization, which could be useful chemical tools for studying CK2's biological roles and potentially be used as therapeutic agents. Importantly, this supports alternative, non-ATP-competitive approaches of achieving CK2 inhibition proposed to afford greater specificity and generate novel drug entities.22 This has been exemplified by the discovery of various chemical disruptors of $\alpha / \beta$ interaction, $\mathrm{CK} 2 \beta$ antagonists, substrate-targeted inhibitor and allosteric inhibitor.27-35 In particular, significant efforts by various groups to develop $\alpha / \beta$ disruptors to interfere with CK2 function highlights the transient character of the $\alpha / \beta$ interaction, as demonstrated by native MS. In our native MS experiments, the observation at intermediate ionic strength (0.30-0.60 M ammonium acetate) of the heterotrimeric CK2 monomer, $\left(a_{1} / \beta_{2}\right)_{1}$ likely represents the outcome of CK2 subunit dissociation from asymmetric trimeric CK2 rings as predicted by PISA, 23 suggesting that CK2 oligomerization occurs via the circular trimeric assembly. However, further elucidation and characterization of the conformational state of CK2 oligomers by IMS-MS and HDX-MS clarified that CK2 oligomerization occurs via both the circular and linear assemblies.

\section{Methods}

Full details of CK2 expression and purification, and mass spectrometric experiments are provided in the Supporting Information online.

\section{Supplementary Material}

Refer to Web version on PubMed Central for supplementary material.

\section{Acknowledgments}

This research was supported by the Wellcome Trust Strategic Award (090340/Z/09/Z), the Agency for Science Technology and Research (A*STAR) Singapore (PhD sponsorship, W.G.S.), the Croucher Foundation and the Cambridge Overseas Trust (Croucher Cambridge International Scholarship, D.S.-H.C). Additional data related to 
this publication can be accessed at the University of Cambridge data repository (https://www.repository.cam.ac.uk/ handle/1810/253341).

\section{References}

(1). Litchfield DW. Protein kinase CK2: structure, regulation and role in cellular decisions of life and death. Biochem J. 2003; 369:1-15. [PubMed: 12396231]

(2). Guerra B. Protein kinase CK2 and its role in cellular proliferation, development and pathology. Electrophoresis. 1999; 20:391-408. [PubMed: 10197447]

(3). Meggio F, Pinna LA. One-thousand-and-one substrates of protein kinase CK2? FASEB J. 2003; 17:349-368. [PubMed: 12631575]

(4). Niefind K, Guerra B, Ermakowa I, Issinger OG. Crystal structure of human protein kinase CK2: insights into basic properties of the CK2 holoenzyme. EMBO J. 2001; 20:5320-5331. [PubMed: 11574463]

(5). Niefind K, Guerra B, Pinna LA, Issinger OG, Schomburg D. Crystal structure of the catalytic subunit of protein kinase CK2 from Zea mays at $2.1 \AA$ A resolution. EMBO J. 1998; 17:24512462. [PubMed: 9564028]

(6). Grankowski N, Boldyreff B, Issinger OG. Isolation and characterization of recombinant human casein kinase II subunits alpha and beta from bacteria. Eur J Biochem. 1991; 198:25-30. [PubMed: 2040287]

(7). Raaf J, Brunstein E, Issinger O, Niefind K. The interaction of CK2 $\alpha$ and CK2 $\beta$, the subunits of protein kinase $\mathrm{CK} 2$, requires $\mathrm{CK} 2 \beta$ in a preformed conformation and is enthalpically driven. Protein Sci. 2008; 17:2180-2186. [PubMed: 18824508]

(8). Meggio F, Boldyreff B, Marin O, Marchiori F, Perich JW, Issinger OG, Pinna LA. The effect of polylysine on casein-kinase-2 activity is influenced by both the structure of the protein/peptide substrates and the subunit composition of the enzyme. Eur J Biochem. 1992; 205:939-945. [PubMed: 1577011]

(9). Rodriguez FA, Contreras C, Bolanos-Garcia V, Allende JE. Protein kinase CK2 as an ectokinase: The role of the regulatory CK2 $\beta$ subunit. Proc Natl Acad Sci. 2008; 105:5693-5698. [PubMed: 18391191]

(10). Poole A, Poore T, Bandhakavi S, McCann RO, Hanna DE, Glover CV. A global view of CK2 function and regulation. Mol Cell Biochem. 2005; 274:163-170. [PubMed: 16342414]

(11). Glover CV. A filamentous form of Drosophila casein kinase II. J Biol Chem. 1986; 261:1434914354. [PubMed: 3095319]

(12). Mamrack MD. Stimulation of enzymatic activity in filament preparations of casein kinase II by polylysine, melittin, and spermine. Mol Cell Biochem. 1989; 85:147-157. [PubMed: 2725485]

(13). Valero E, De Bonis S, Filhol O, Wade RH, Langowski J, Chambaz EM, Cochet C. Quaternary structure of casein kinase 2. Characterization of multiple oligomeric states and relation with its catalytic activity. J Biol Chem. 1995; 270:8345-8352. [PubMed: 7713943]

(14). Niefind K, Issinger O-G. Primary and secondary interactions between CK2alpha and CK2beta lead to ring-like structures in the crystals of the CK2 holoenzyme. Mol Cell Biochem. 2005; 274:3-14. [PubMed: 16335523]

(15). Schnitzler A, Olsen BB, Issinger OG, Niefind K. The protein kinase CK2(Andante) holoenzyme structure supports proposed models of autoregulation and trans-autophosphorylation. J Mol Biol. 2014; 426:1871-1882. [PubMed: 24594356]

(16). Lolli G, Pinna LA, Battistutta R. Structural determinants of protein kinase CK2 regulation by autoinhibitory polymerization. ACS Chem Biol. 2012; 7:1158-1163. [PubMed: 22506723]

(17). Lolli G, Ranchio A, Battistutta R. Active form of the protein kinase CK2 a $2 \beta 2$ holoenzyme is a strong complex with symmetric architecture. ACS Chem Biol. 2014; 9:366-371. [PubMed: 24175891]

(18). Chantalat L, Leroy D, Filhol O, Nueda A, Benitez MJ, Chambaz EM, Cochet C, Dideberg O. Crystal structure of the human protein kinase CK2 regulatory subunit reveals its zinc fingermediated dimerization. EMBO J. 1999; 18:2930-2940. [PubMed: 10357806] 
(19). Rekha N, Srinivasan N. Structural basis of regulation and substrate specificity of protein kinase CK2 deduced from the modeling of protein-protein interactions. BMC Struct Biol. 2003; 3:4. [PubMed: 12740046]

(20). Pagano MA, Sarno S, Poletto G, Cozza G, Pinna LA, Meggio F. Autophosphorylation at the regulatory $\beta$ subunit reflects the supramolecular organization of protein kinase CK2. Mol Cell Biochem. 2005; 274:23-29. [PubMed: 16335525]

(21). Lüscher B, Litchfield DW. Biosynthesis of casein kinase II in lymphoid cell lines. Eur J Biochem. 1994; 220:521-526. [PubMed: 8125110]

(22). Prudent R, Cochet C. New protein kinase CK2 inhibitors: jumping out of the catalytic box. Chem Biol. 2009; 16:112-120. [PubMed: 19246001]

(23). Krissinel E, Henrick K. Inference of macromolecular assemblies from crystalline state. J Mol Biol. 2007; 372:774-797. [PubMed: 17681537]

(24). Martel V, Filhol O, Nueda A, Cochet C. Dynamic localization/association of protein kinase CK2 subunits in living cells: a role in its cellular regulation? Ann N Y Acad Sci. 2002; 973:272-277. [PubMed: 12485876]

(25). Sarno S, Vaglio P, Marin O, Meggio F, Issinger OG, Pinna LA. Basic residues in the 74-83 and 191-198 segments of protein kinase CK2 catalytic subunit are implicated in negative but not in positive regulation by the beta-subunit. Eur J Biochem. 1997; 248:290-295. [PubMed: 9346280]

(26). Hübner GM, Larsen JN, Guerra B, Niefind K, Vrecl M, Issinger OG. Evidence for aggregation of protein kinase CK2 in the cell: a novel strategy for studying CK2 holoenzyme interaction by BRET2. Mol Cell Biochem. 2014; 397:285-293. [PubMed: 25148873]

(27). Laudet B, Barette C, Dulery V, Renaudet O, Dumy P, Metz A, Prudent R, Deshiere A, Dideberg $\mathrm{O}$, Filhol O, Cochet C. Structure-based design of small peptide inhibitors of protein kinase CK2 subunit interaction. Biochem J. 2007; 408:363-373. [PubMed: 17714077]

(28). Laudet B, Moucadel V, Prudent R, Filhol O, Wong Y-S, Royer D, Cochet C. Identification of chemical inhibitors of protein-kinase CK2 subunit interaction. Mol Cell Biochem. 2008; 316:6369. [PubMed: 18553057]

(29). Raaf J, Brunstein E, Issinger O-G, Niefind K. The CK2a/CK2 $\beta$ interface of human protein kinase CK2 harbors a binding pocket for small molecules. Chem Biol. 2008; 15:111-117. [PubMed: 18291315]

(30). Raaf J, Guerra B, Neundorf I, Bopp B, Issinger O-G, Jose J, Pietsch M, Niefind K. First structure of protein kinase CK2 catalytic subunit with an effective CK2 $\beta$-competitive ligand. ACS Chem Biol. 2013; 8:901-907. [PubMed: 23474121]

(31). Hochscherf J, Lindenblatt D, Steinkrüger M, Yoo E, Ulucan Ö, Herzig S, Issinger OG, Helms V, Götz C, Neundorf I, Niefind K, et al. Development of a high-throughput screening-compatible assay to identify inhibitors of the CK2a/CK2 $\beta$ interaction. Anal Biochem. 2015; 468:4-14. [PubMed: 25233003]

(32). Prudent R, Moucadel V, Laudet B, Barette C, Lafanechère L, Hasenknopf B, Li J, Bareyt S, Lacôte E, Thorimbert S, Malacria M, et al. Identification of polyoxometalates as nanomolar noncompetitive inhibitors of protein kinase CK2. Chem Biol. 2008; 15:683-692. [PubMed: 18635005]

(33). Martel V, Filhol O, Colas P, Cochet C. p53-dependent inhibition of mammalian cell survival by a genetically selected peptide aptamer that targets the regulatory subunit of protein kinase CK2. Oncogene. 2006; 25:7343-7353. [PubMed: 16751801]

(34). Perea SE, Reyes O, Puchades Y, Mendoza O, Vispo NS, Torrens I, Santos A, Silva R, Acevedo B, Lopez E, Falcon V, et al. Antitumor effect of a novel proapoptotic peptide that impairs the phosphorylation by the protein kinase 2 (casein kinase 2). Cancer Res. 2004; 64:7127-7129. [PubMed: 15466209]

(35). Perea SE, Reyes O, Baladron I, Perera Y, Farina H, Gil J, Rodriguez A, Bacardi D, Marcelo JL, Cosme K, Cruz M, et al. CIGB-300, a novel proapoptotic peptide that impairs the CK2 phosphorylation and exhibits anticancer properties both in vitro and in vivo. Mol Cell Biochem. 2008; 316:163-167. [PubMed: 18575815] 
a

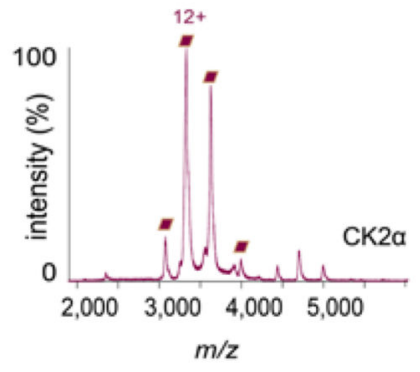

$\mathrm{C}$

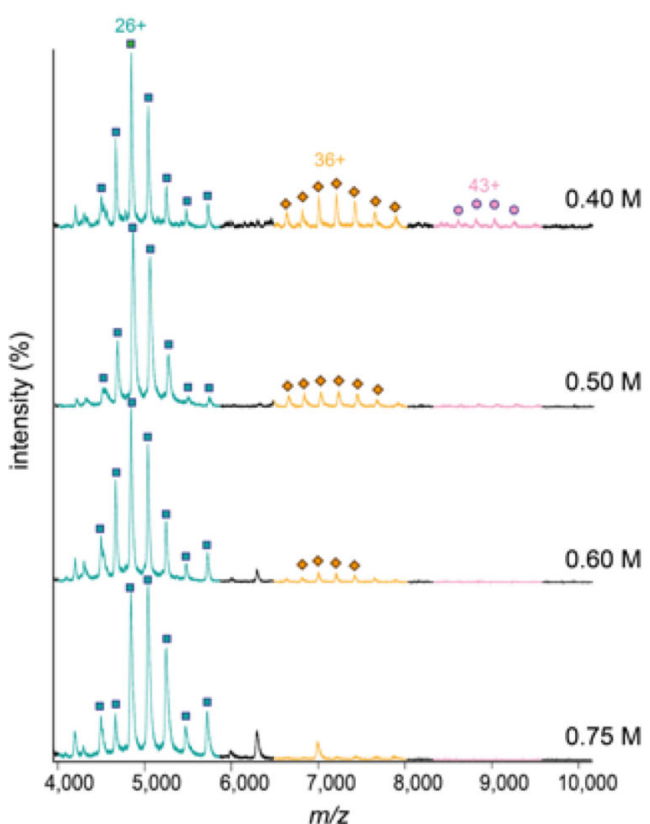

b

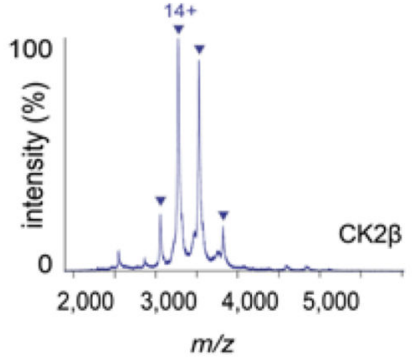

Excess CK2 $\beta$

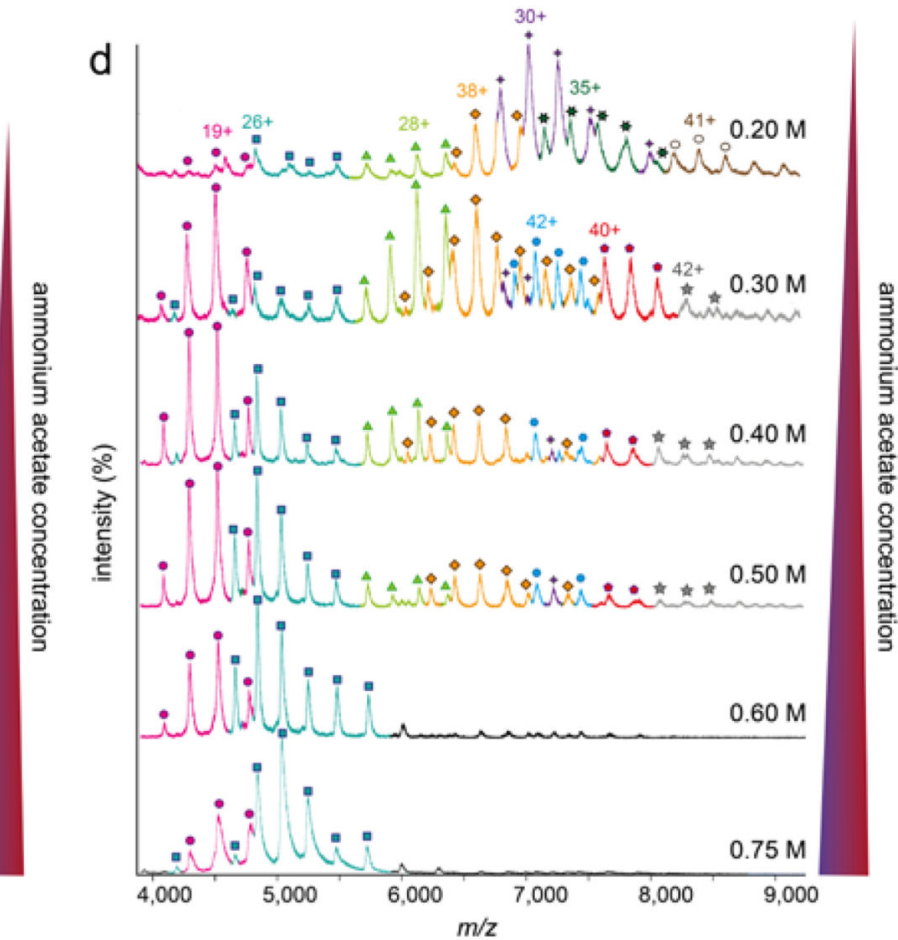

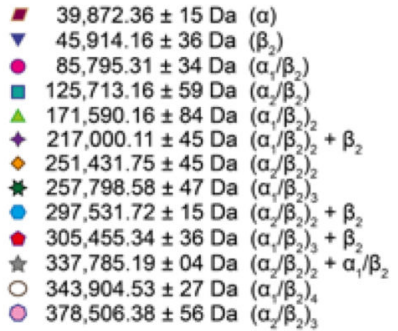

Figure 1. Native mass spectra of CK2 $a, \mathrm{CK} 2 \beta$, and CK2 acquired by nESI-MS under different experimental conditions.

(a) Spectra of monomeric CK2 $a(5 \mu \mathrm{M})$ in $0.50 \mathrm{M}$ ammonium acetate, showing four charge states. (b) Spectra of dimeric CK2 $\beta(5 \mu \mathrm{M})$ in $0.50 \mathrm{M}$ ammonium acetate, with four charged states recorded. (c), (d) Spectra of CK2 complexes and oligomers $(10 \mu \mathrm{M})$ in solutions of various ionic strength (0.40-0.75 M ammonium acetate), showing only species with $\mathrm{m} / \mathrm{Z}$ > 4,000, when 2-fold molar excess of CK2 $a$ and 1.2-fold molar excess of CK2 $\beta$ was used, respectively. Charge states are colored and indicated with symbols, each representing a 
different species. The observed mass and identity of each species are indicated beside the symbols. Only the main charge state of each species is indicated in the spectra. 

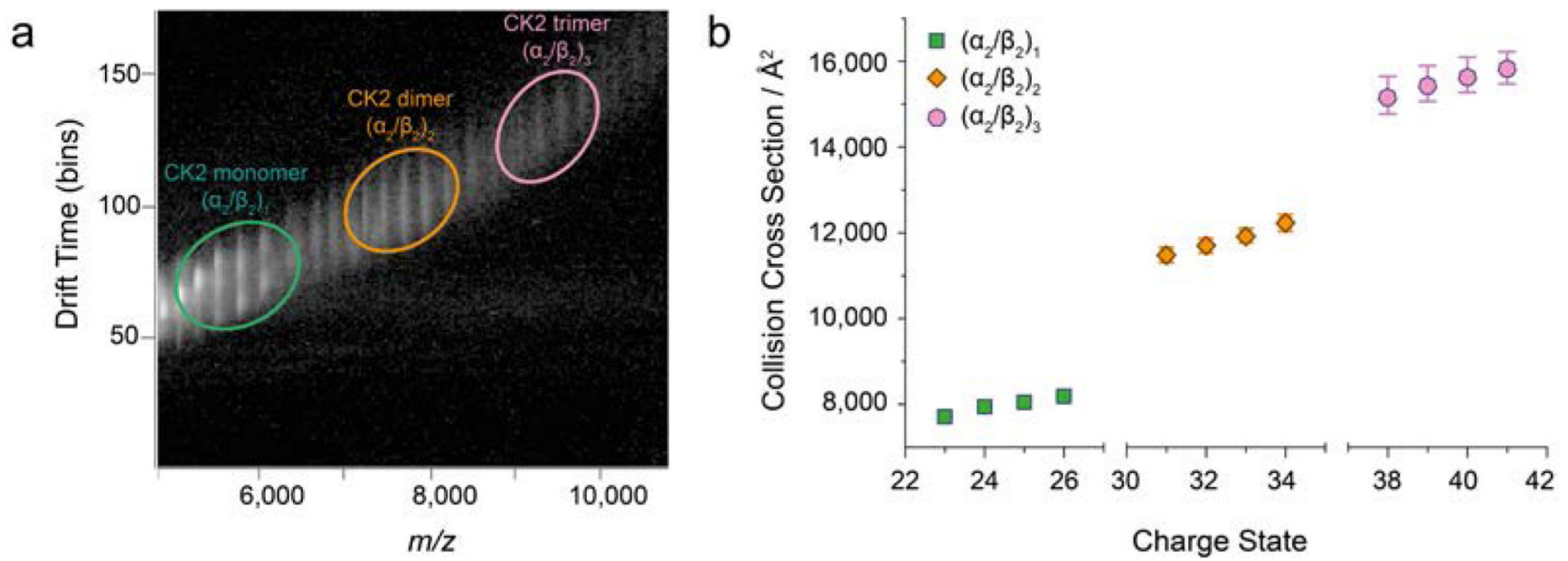

Figure 2. Ion mobility spectrometry-mass spectrometry of the monomeric, dimeric and trimeric CK2 heterotetramer.

(a) Contour plot of drift time versus $\mathrm{m} / z$ for CK2 $(10 \mu \mathrm{M})$ in $0.40 \mathrm{M}$ ammonium acetate. Monomeric, dimeric, and trimeric CK2 species are indicated with green, orange, and pink ellipses, respectively. (b) Drift times from (a) converted into CCS and then plotted against charge state. 
a

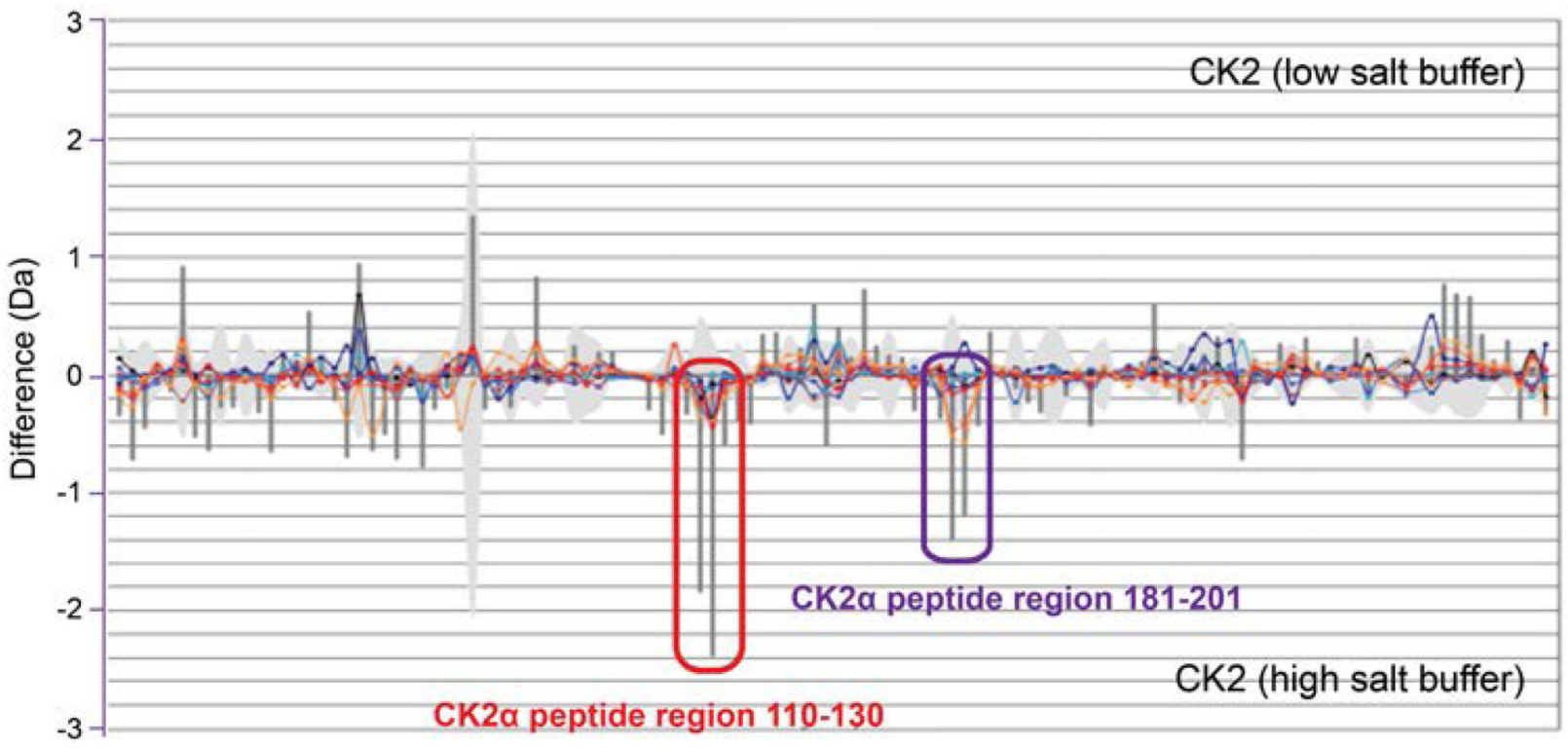

b

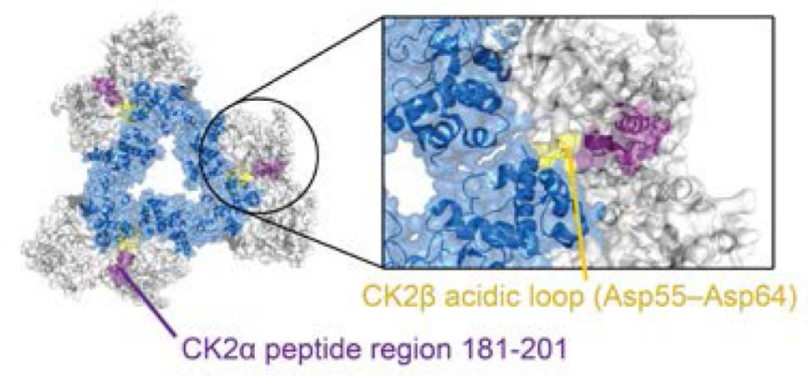

C

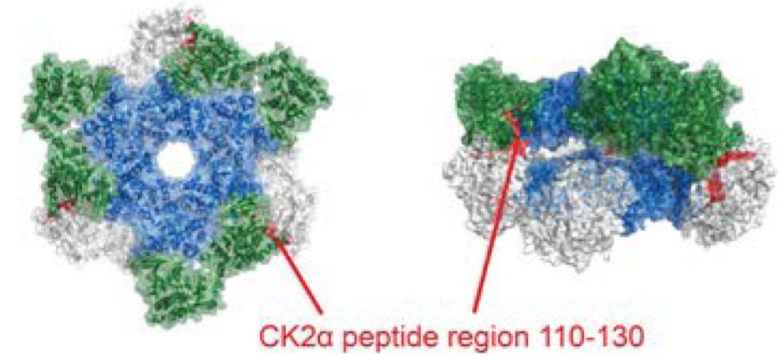

d

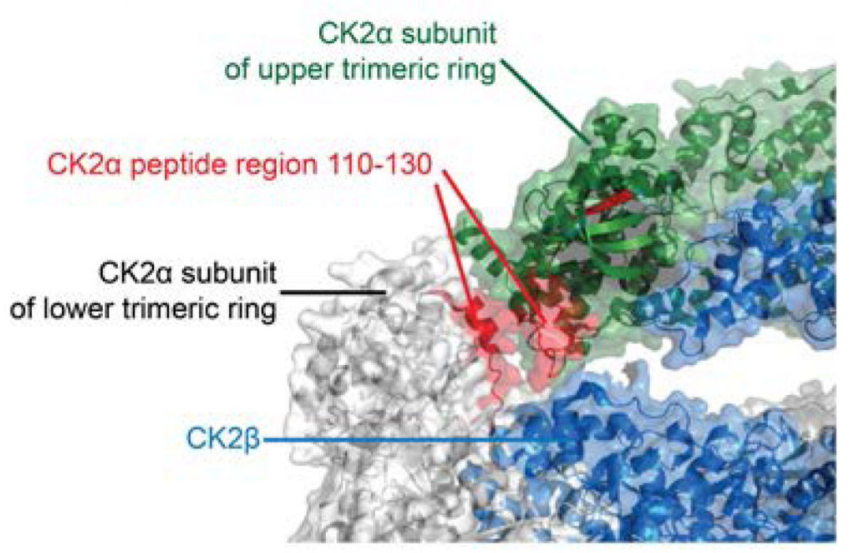

Figure 3. HDX-MS experiment of CK2 complexes and crystal structure of the circular CK2 assembly, onto which HDX-MS data are mapped.

(a) Butterfly plot of difference in deuterium uptake of CK2 $a$ in low $(0.20 \mathrm{M} \mathrm{NaCl})$ and high salt $(0.75 \mathrm{M} \mathrm{NaCl})$ conditions. The $y$-axis indicates the difference in deuterium uptake (Da), while the $x$-axis denotes detected CK2 $a$ peptides arranged in order of increasing residue number from the $\mathrm{N}$-terminus. Vertical grey lines represent the total difference of each peptide summed over all time points of the HDX-MS experiment. Colored lines show uptake difference from 0.5-180 min labeling time. (b) Ring conformation of trimeric CK214 (PDB: 1JWH4) with an inset showing a close-up view of the interaction between the peptide region 181-201 (purple) on CK2 $a$, which experienced decreased deuterium exchange in low salt with the acidic loop of CK2 $\beta$ (yellow). (c) Top and side views of two stacked trimeric rings (PDB: 4DGL16). The peptide region 110-130 of CK2 $a$, which experienced decreased 
deuterium exchange in low salt buffer, is shown in red. (d) A close-up view of the trimertrimer interaction interface involving peptide region 110-130 (red) on CK2 $a$ as a result of stacked trimeric ring formation. CK2 $a$ subunits are colored white or green, while CK2 $\beta$ subunits are colored blue. 
a

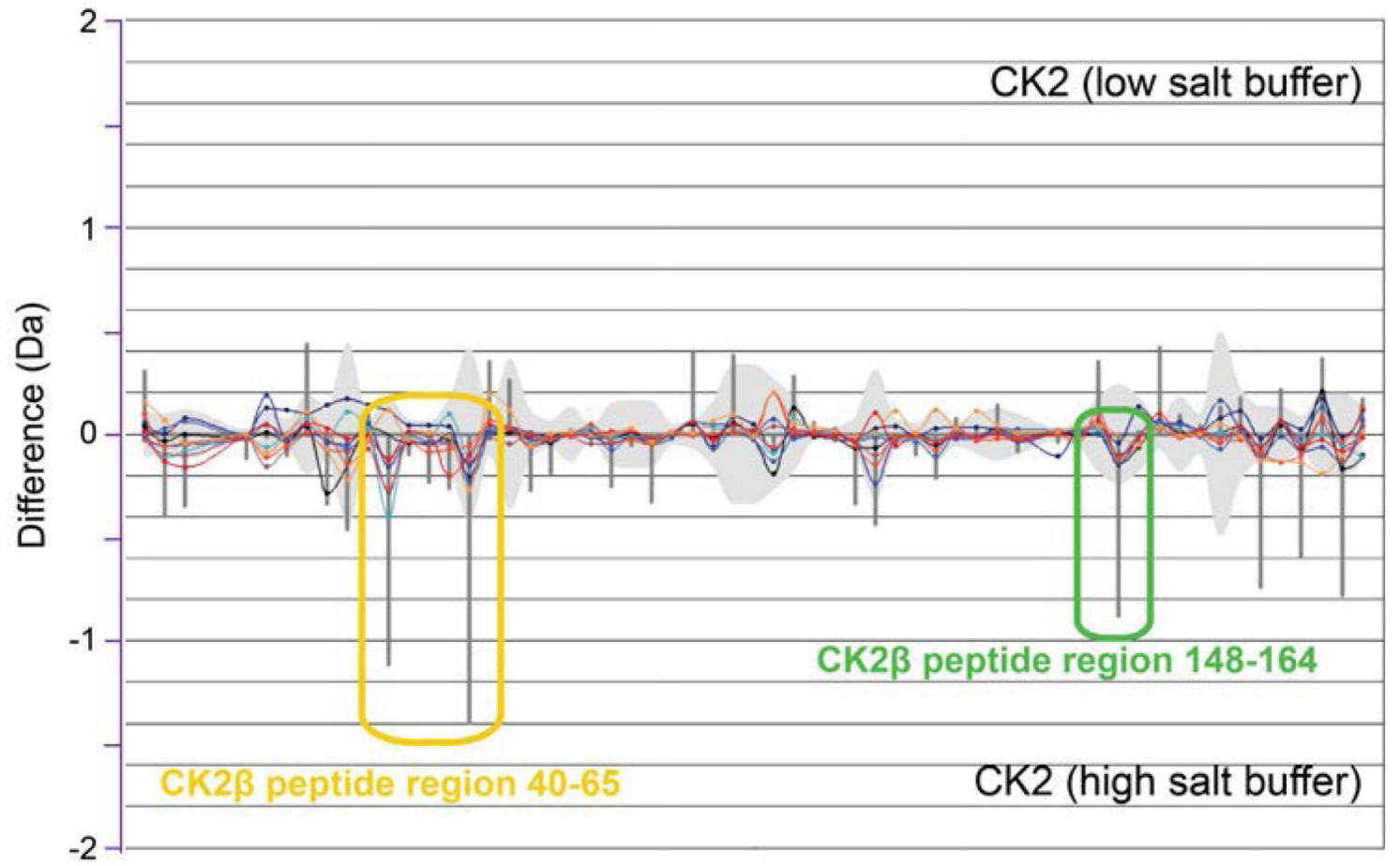

b

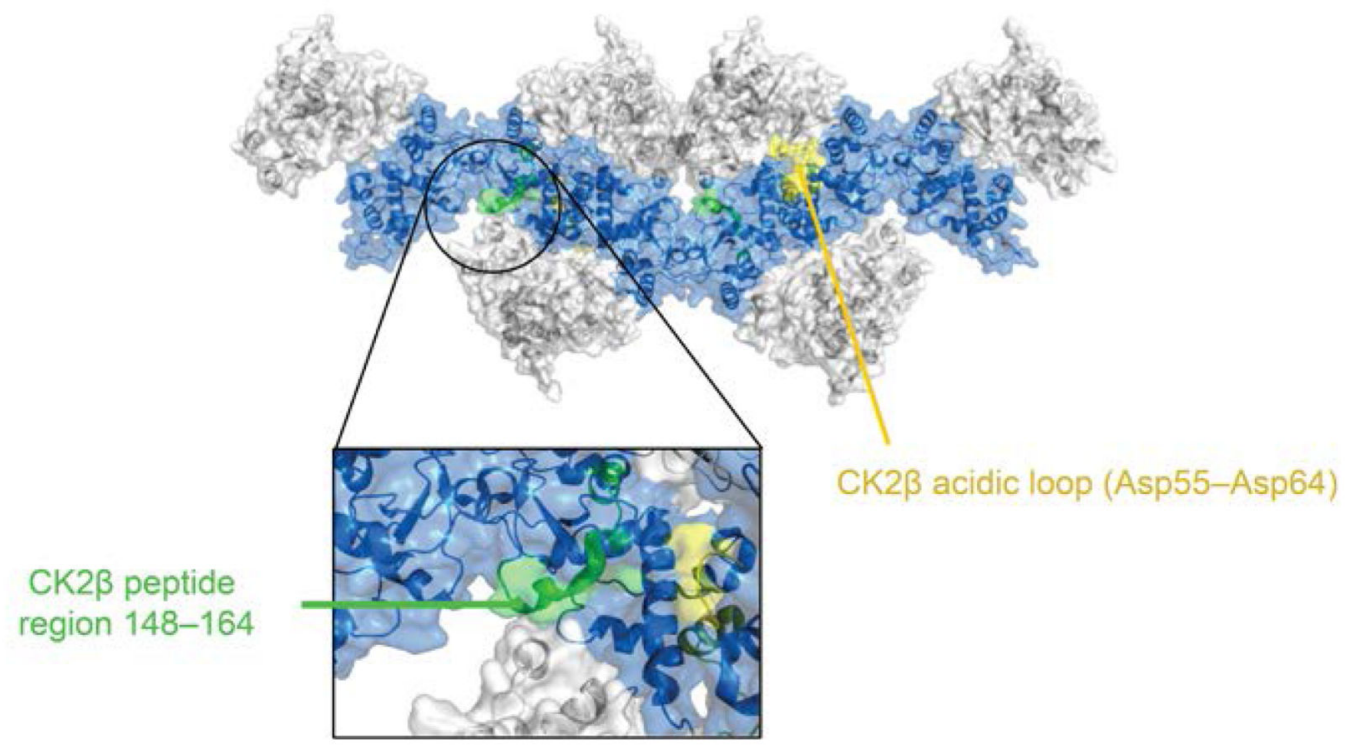

Figure 4. HDX-MS experiment of CK2 complexes and crystal structure of the linear CK2 assembly, onto which HDX-MS data are mapped.

(a) Butterfly plot of difference in deuterium uptake of $\mathrm{CK} 2 \beta$ in low $(0.20 \mathrm{M} \mathrm{NaCl})$ and high salt $(0.75 \mathrm{M} \mathrm{NaCl})$ conditions. The $y$-axis indicates the difference in deuterium uptake (Da), while the $x$-axis denotes detected CK $2 \beta$ peptides arranged in order of increasing residue number from the $\mathrm{N}$-terminus. Vertical grey lines represent the total difference of each peptide summed over all time points of the HDX-MS experiment. Colored lines show uptake difference from 0.5-180 min labeling time. (b) Linear conformation of CK2 (PDB: 4NH115) with an inset showing a close-up view of the interaction between the peptide 
region 148-164 (green) on CK2 $\beta$ and a CK2 $a$ subunit (white) from a neighboring holoenzyme. CK2 $a$ and CK2 $\beta$ subunits are colored white and blue, respectively. 


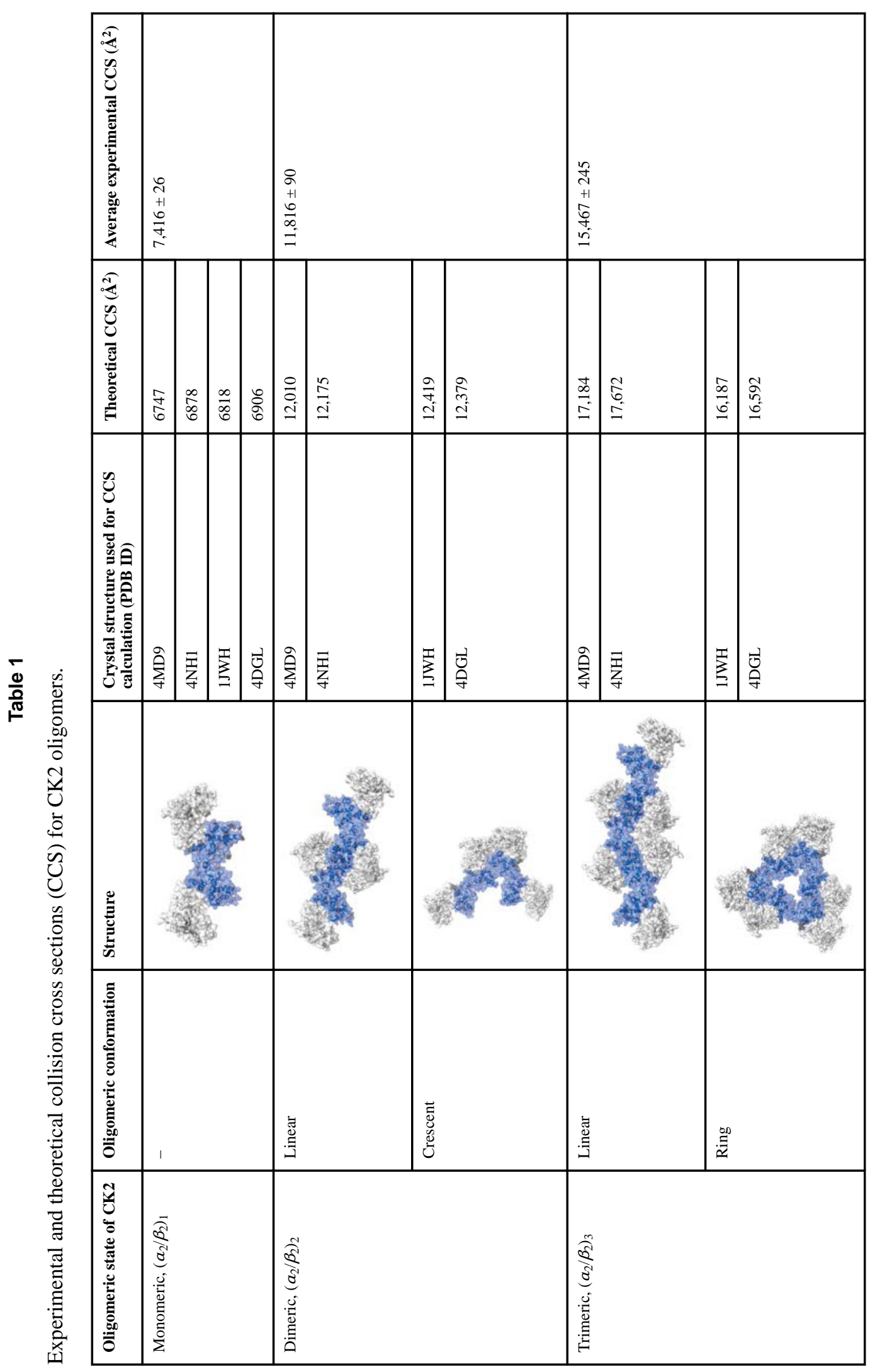

ACS Chem Biol. Author manuscript; available in PMC 2018 March 26. 